
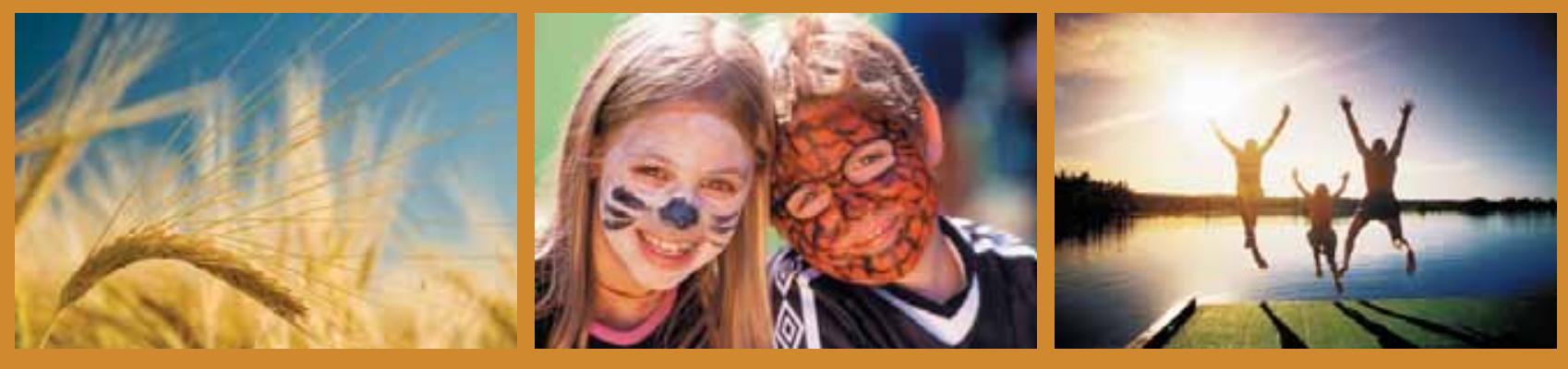

\title{
Congress at a Glance
}

TuESDAY, JULY 17, 2007

8:30 am - 4:00 pm

CAO Council M eeting

7:00 pm - 10:00 pm

CAO Council \& Congress Committee Social

Wednesday, JuLY 18, 2007

10:00 am - 7:30 pm Congress Registration Desk Open (TCU Place)

8:30 am - 9:30 am

Golf Registration (Willows Golf \& Country Club)

10:00 am - 6:00 pm

CAO President's Cup Golf Tournament

7:30 pm - 10:00 pm

President's Welcoming Reception (Top of the Inn)

\section{ThuRSDAY, July 19, 2007}

7:30 am - 4:30 pm

7:30 am - 8:15 am

$7: 45 \mathrm{am}-4: 45 \mathrm{pm}$

8:15 am - 10:15 am

10:00 am - 4:45 pm

10:00 am - 5:00 pm

$10: 30 \mathrm{am}-12: 00 \mathrm{pm}$

$12: 00 \mathrm{pm}-1: 00 \mathrm{pm}$

1:00 pm - 4:15 pm

6:00 pm -

Registration Desk Open (TCU Place)

Breakfast (TCU Place)

Children's Program Open (Sheraton Hotel)

CE Sessions, OD's \& Assistants (TCU Place)

Companion / Teen Program

Alternate Golf \& Tours (Dunes / M oon Lake)

CE Sessions, OD's \& Assistants (TCU Place)

Lunch

CE Sessions, OD's \& Assistants (TCU Place) Official CAO Congress Opening Ceremonies (Western Development Museum) 

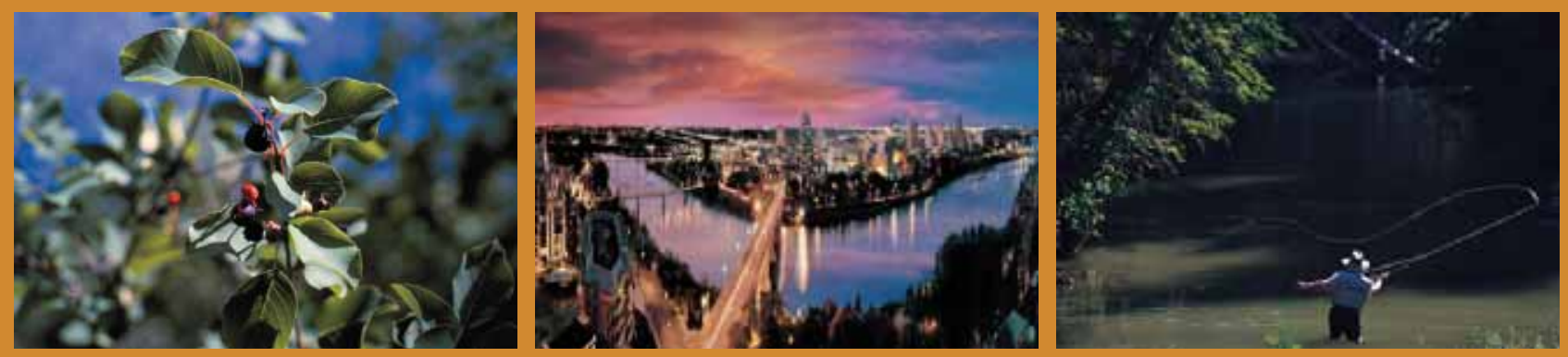

FrIDAY, JULY 20, 2007

7:30 am - 4:30 pm

7:30 am - 8:15 am

7:45 am - 4:45 pm

8:15 am - 10: $15 \mathrm{am}$

10:00 am - 4:30 pm

$10: 30 \mathrm{am}-11: 30 \mathrm{am}$

$12: 00 \mathrm{pm}-1: 30 \mathrm{pm}$

$11: 30 \mathrm{am}-4: 00 \mathrm{pm}$

5:00 pm - 7:00 pm

7:00 pm - 10:00 pm
Registration Desk Open (TCU Place)

Breakfast (TCU Place)

Children's Program Open (Sheraton Hotel)

CE Sessions, OD's \& Assistants (TCU Place)

Companion / Teen Program

CE Sessions, OD's \& Assistants (TCU Place)

Past President's Lunch

OPTOFAIR \& Lunch (TCU Place)

School of Optometry, UW Reception (location TBD)

Class Reunions (on own)

\section{SAturday, July 21, 2007}

7:30 am - 4:00 pm

7:30 am - 8:15 am

7:45 am - 10:00 pm

8:15 am - 10:15 am

10:00 am - 4:00 pm

10:30 am- 12:00 pm

$12: 00 \mathrm{pm}-1: 00 \mathrm{pm}$

$1: 00 \mathrm{pm}-4: 00 \mathrm{pm}$

4:00 pm - 10:00 pm

5:30 pm - 7:00 pm

6:00 pm - 7:00 pm
Registration Desk Open (TCU Place)

Breakfast (TCU Place)

Children's Program (Sheraton Hotel)

CE Sessions, OD's \& Assistants (TCU Place)

Companions / Teen Programs

CE Sessions, OD's \& Assistants (TCU Place)

Lunch

CAO Business M eeting (TCU Place)

Children's / Teen Evening Program (Sheraton Hotel) VIP, Champagne Reception (TCU Place)

President's Reception, Banquet \& Ball (TCU Place)

\section{SUNDAY, JuLY 22, 2007}

Departures

Ad Hoc M eetings 